11 Pushpakom, R., et al., American Review of Respiratory Diseases, 1970 $102,778$.

Talamo, R. C., Allen, J. D., Kahan, M. G., and Austen, K. F., New England fournal of Medicine, 1968, 278, 345.

12 Guenter, C. A., Welch, M. H., Russell, R. T., Hyde, R. M., and Hammarstein, J. F., Archives of Internal Medicine, 1968, 122, 254.

14 Townley, R. 'G., Ryning, F., Lynch, H., and Brody, A. F., fournal of the American Medical Association, 1970, 214, 325.

1s Stein, P. D., Leu, J. D., Welch, M. H., and Guenter, C. A., Circulation, $1971,43,227$.

10 Schleusener, A., Talamo, R. C., Paré, J. A. P., and Thurlbeck, W. M., American Review of Respiratory Diseases, $1968,98,692$.

17 Tarkoff, M. P., Kueppers, F., and Miller, W. F., American fournal of

Medicine, 1968, 45, 220.
18 Heard, B. E., Thorax, 1959, 14, 58.

10 Heard, B. E., Thorax, 1959, 14, 58. $1961,31,279$.

20 Gaziano, D., Seaton, A., and Ogilvie, C., British Medical fournal, 1970, 2, 330.

21 Anthonisen, N. R., Bass, H., Oriel, A., Place, R. E. G., and Bates, D. V. Clinical Science, 1968, 35, 495.

"2 Ogilvie, C., Thorax, 1967, 22, 286.

23 West, J. B., fournal of Applied Physiology, 1962, 17, 893.

\section{Varieties of Smoke}

One of the recommendations made by the Royal College of Physicians ${ }^{12}$ in its report on smoking and health published earlier this year concerned the chemical content of cigarettes. The tar and nicotine content of all marketed brands of cigarettes should be published, it said, and should be printed on the packets. A committee under the aegis of the Department of Health is at present examining the possibility of doing this. Last week the Consumer Association's magazine Which? ${ }^{3}$ anticipated Government action by publishing a table showing the amount of tar and nicotine per cigarette for a number of brands and for some small cigars.

If a person could choose to smoke a brand of cigarettes known to have a low content of tar and nicotine he might be able to reduce the risk of ill health from his habit. At least common sense suggests this and the royal college's report declares it. But is it true? For what needs to be considered is the term "content." The Which? report does not state how the amounts of tar and nicotine it lists in milligrams per cigarette were extracted, but we are informed that a smoking machine was used. The presence or absence of a filter would make a difference to the yield, though the cigarette stub has a filtering effect and filters themselves vary in efficiency. In fact some untipped cigarettes were found to yield less tar and nicotine than some with filter tips. But the difficulty remains of how to interpret any published figures in terms of comparative yield of tar and nicotine by different brands of cigarette to the mouth and respiratory system of the smoker.

The doubt about the meaning of such figures in practical terms derives from the variation in smoking behaviour of people using different kinds of cigarettes. People do not smoke like machines. Heather Ashton and D. W. Watson ${ }^{4}$ showed that smokers of low-nicotine cigarettes had a higher frequency of puffing than smokers of highnicotine cigarettes and drew into their mouths nearly the same amount of nicotine. Smokers seemed to adjust their technique to provide themselves with the dose of nicotine that they needed to satisfy their desire for it.

But if the Which? figures are difficult to interpret the advice it gives its readers is incontestable: "Giving up smoking altogether is the one course open to smokers which is of real benefit." The Department of Health might do better to hammer that home than authorize what would inevitably be a considerable expenditure of money, skill, and time on analysing the tar and nicotine content of cigarettes or tobacco in any form. It is questionable whether such figures, however obtained and presented, could offer a basis for a "healthy" choice of cigarette.

1 Royal College of Physicians of London, Smoking and Health Now. London, Pitman, 1971.

2 British Medical fournal, 1971, 1, 61.

3 Consumers' Association, Which?, September 1971.

4 Ashton, H., and Watson, D. W., British Medical fournal, 1970, 3, 679.

\section{Combined Virus Vaccines}

The decline in the incidence of measles in the United States and Great Britain since the use of live attenuated measles vaccine suggests that the disease could be brought under control, or even eradicated, by vaccination. The fact that this has not yet been achieved is largely a problem of getting vaccine to the susceptible population. Attenuated vaccines against rubella have also been developed and are in use, and more recently a mumps vaccine has joined the ever growing list of immunological products. If all these vaccines were administered separately the number of visits to doctors and clinics for inoculations would be intolerable. The use of combined vaccines or the simultaneous administration of vaccines, or both, offers some solution to the problem. Combinations of inactivated vaccines can be prepared without much difficulty, and many, such as T.A.B. vaccine and triple vaccine (diphtheria-pertussis-tetanus prophylactic) have been in use for some years.

Live virus vaccines can also be prepared as combined products, and they have a specially important role in developing countries, for they can be given not only as combined vaccines against severe epidemic diseases but by less costly forms of administration, such as the jet injector. In several African countries many millions of individuals have been immunized with combined smallpox and yellow fever vaccines. Combined attenuated vaccines given by jet injector have also been used against measles, smallpox, and yellow fever with some success. 1

Now that attenuated vaccines are available against measles, rubella, and mumps, the question arises of who shall be immunized and at what age. Despite the reduction in mortality from measles in the past 50 years in the developed countries, the general incidence and morbidity from the disease (before immunization came in) remained high. Protection is required because of the complications, particularly of the central nervous system and respiratory tract. As measles is a disease of late infancy and early childhood, immunization should be carried out in young children. Rubella usually occurs later in childhood or adolescence and is of serious consequence, owing to the risk to the fetus, only when a susceptible woman contracts the disease in early pregnancy. There are at present two schools of thought about the best way of protecting women of childbearing age. Either large-scale immunization of children of both sexes can be offered before the age of childbearing in an attempt to reduce the natural reservoir of infection in children. Or, as recommended in Britain, selective immunization can be offered to girls before the age of childbearing and also to adult women known to be susceptible, and provided the risk of pregnancy is avoided. The difference in these approaches is based mainly on present uncertainties about the duration 
of immunity from rubella vaccines. And the same uncertainty applies to mumps. This is another common infectious disease of childhood which may, like rubella, occur also in the adult. Among the complications of the disease the most frequent are lesions of the central nervous system, but fortunately most of these cases are of aseptic meningitis and the prognosis is usually good, in contrast to the severe encephalitis associated with measles. Orchitis, another well-known complication, is rarely bilateral, and the belief that it may lead to sterility is based more on fear and pain than on fact.

M. R. Hilleman and his colleagues ${ }^{2} 3$ at West Point, Pennsylvania, have shown that mumps vaccine prepared in duck embryo cell cultures was immunogenic, generally free from reactions, and conferred protection when an outbreak of mumps occurred shortly after administration of the vaccine. They have now studied combinations of measlesrubella-mumps ${ }^{4}$ and rubella-mumps vaccines. ${ }^{5}$ Studies on several hundred children who initially lacked antibody to all three viruses showed seroconversion rates of between 94 and 99\% against all antigenic components. The antibody levels were much the same as when the vaccines were administered separately. These studies indicate that simultaneous administration of combined vaccines is not accomoanied by interference of one vaccine with the other, and there was no increase in clinical reactions owing to the vaccines being given together. If a decision is taken to protect children, either individually or collectively, against these diseases, it is useful to know that the vaccines can be combined in a single dose, but it would seem that we need to know more about the long-term protective efficacy of a mumps vaccine before it is adopted as a routine measure for children. In the meantime it would appear to be a useful vaccine for adults who have escaped childhood infection.

' Mever, H. M., Hopps, H. E., Bernheim, B. C., and Douglas, R. D., in First International Conference on Vaccines against Viral and Rickettsial Diseases of Man, Washington, November, 1966, p. 336. Washington, Pan American Health Organization, 1967.

Weibel, R. E., Buynak, E. B., Stokes, J., Whitman, J. E., and Hilleman, M. R., in First International Conference on Vaccines against Viral and Rickettsial Diseases of Man, Washington, November, 1966, p. 430. Wickettsial Diseases of Man, Washington, November,

- Hilleman, M. R., Buynak, E. B., Weibel, R. E., and Stokes, J., New England fournal of Medicine, 1968, 278, 227.

- Buynak, E. B., Weibel, R. E., Whitman, J. E., Stokes, J., and Hilleman, M. R., Fournal of the American Medical Association, 1969, 207, 2259. -Weibel, R. E., et al., fournal of the American Medical Association, 1971, 216, 983.

\section{Malignant Disease and the Nephrotic Syndrome}

From time to time reports appear of patients suffering simultaneously from malignant disease and the nephrotic syndrome. In some the renal condition may be attributable to amyloidosis $^{1}$ or to renal vein thrombosis, ${ }^{2}$ and in others the association may be due to chance. However, J. C. Lee and his colleagues ${ }^{3}$ calculated that the association was much more frequent than would be expected on this basis, and it seems likely that there is some other connexion between the two conditions.

The possibilities would seem to be that both have a common cause, or that the nephrotic syndrome arises as a complication of the neoplasm. The latter hypothesis seems particularly likely in those rare cases in which treatment directed towards the neoplasm results in a remission of the nephrotic syndrome. In 1969 E. G. Cantrell ${ }^{4}$ reported on a patient whose nephrotic syndrome remitted after subtotal gastrectomy for carcinoma of the stomach, and recently $\mathrm{J}$. Plager and L. Stutzman 5 have reviewed reports of Hodgkin's disease associated with the nephrotic syndrome. They discovered ten cases and reported four more of their own. These four cases showed a remarkable temporal correlation between multiple relapses and remissions of the two conditions. In three cases investigations performed in life or at postmortem excluded the possibilities of amyloidosis or renal vein thrombosis, and in any case a relapsing nephrotic syndrome would be a very unusual manifestation of these disorders. Renal tissue was obtained by biopsy in two cases and at necropsy in two. The appearance of the material was reported to be within normal limits, but unfortunately it was not possible to examine it either by immunofluorescent techniques or by electron microscopy. Indeed, the extent of the examination by light microscopy is far from clear. However, many features of the patients' illnesses suggested that the renal lesions were minimal. Plager and Stutzman point out that much of the treatment they prescribed, particularly the steroid and cytostatic drugs, might be expected to benefit the renal disorder directly. But on several occasions remissions of both disorders followed short courses of radiotherapy applied to peripheral lymph nodes, and the authors conclude that the nephrotic syndrome occurring in association with Hodgkin's disease is likely to be due to renal damage induced either directly by a substance produced by the tumour or indirectly via an immunological reaction to the tumour. This could lead either to the formation of circulating antigen-antibody complexes, which might lodge in the kidney, or to renal damage due to cross-reaction of relatively non-specific antitumour antibodies with the glomerular basement membrane.

Hodgkin's disease is notorious for the multiplicity of its systemic manifestations, and it does not seem unreasonable to postulate the production by the tumour of a substance which could alter the permeability of the glomerular basement membrane without producing gross histological changes. On the other hand, glomerulonephritis produced experimentally by circulating antigen-antibody complexes or by antibody to glomerular basement membrane is associated with changes visible on ordinary light microscopy. 6 Thus participation of either of these mechanisms in the three cases having normal renal morphology seems to be rather unlikely. However, perhaps different mechanisms operate in different patients. Two of the patients from reports cited by Plager and Stutzman were said to have membranous nephropathy, and this appears to be the commonest renal lesion when the nephrotic syndrome is associated with carcinomata. ${ }^{3}$

Recently L. W. Loughridge and M. G. Lewis, from the Westminster Hospital, ${ }^{7}$ reported three more cases with malignant disease and the nephrotic syndrome. One of these, a man with carcinoma of the bronchus, had a membranous nephropathy, which was studied by immunofluorescent techniques. Deposits of IgG and IgM were found on the glomerular basement membrane. They had a "lumpy" appearance characteristic of that seen after the deposition of antigen-antibody complexes and unlike the "linear" appearance characteristic of nephritis produced by anti-kidney serum. Subsequently ${ }^{8}$ further (necropsy) material from the same patient was examined, and identical antigens were detected in an extract of the tumour and an eluate from the glomeruli.

Plager and Stutzman conclude their article by comparing their patients to the New Zealand black mice. Animals of this species develop a complex illness with glomerulonephritis and have a high incidence of lymphomata. A murine 